\title{
IMPROVED INTERFERENCE CANCELLATION IN SYNTHESIS ARRAY RADIO ASTRONOMY USING AUXILIARY ANTENNAS
}

\author{
Brian D. Jeffs, Karl Warnick and Lisha Li \\ Brigham Young University, Department of Electrical and Computer Engineering \\ Provo, UT 84602. bjeffs@ee.byu.edu, warnick@ee.byu.edu, lisha@ee.byu.edu
}

\begin{abstract}
Spatial filtering and subspace projection methods have been proposed for removing interference signals at radio astronomy (RA) imaging arrays [1][2]. Given the fact that RA signal levels are usually below noise levels, and since high gain antennas significantly reduce the interference to noise level at antenna feeds, it is often difficult to form the accurate interference signal parameter estimates. By adding a few (1-3) low gain "auxiliary" antennas to an imaging array, it is possible to overcome this problem. Using low cost auxiliaries with an existing array can improve interference rejection by tens of decibels. New extensions to subspace projection spatial filtering methods are presented, along with simulated results for performance comparison.
\end{abstract}

\section{INTRODUCTION}

This paper discusses how using a few low-gain auxiliary antennas as part of a radio astronomy (RA) imaging array makes it possible to obtain outstanding rejection of interfering sources. We are particularly interested in canceling interference from orbital satellite downlink signals such as from GLONASS and IRIDIUM. These very strong interferers affect critical observation spectral bands used in radio astronomy [3]. For example, the Russian GLONASS positioning satellite signal produces strong spectral sidelobes that extend through the important hydroxyl ion $(\mathrm{OH})$ emission band.

In many practical scenarios with satellite interference, we have found poor cancellation performance when using existing array processing and subspace projection methods. Shallow, unstable beamforming nulls and poor interferer subspace estimates are common. This is usually due to the relatively low interference-tosignal-plus-noise ratio (ISNR) at antenna feeds of the high gain antennas used in imaging arrays. This problem occurs even when interfering sources have flux densities which are many tens of decibels higher than the desired signal, because of the low sidelobe response for the high gain antennas. It must be remembered that in RA, signals of interest are usually well below the noise floor, so an interference level at antenna feeds which is much higher than the signal may not be significantly higher than the noise. In this case it is difficult to form the accurate interference signal parameter estimates necessary for high performance adaptive cancellation or subspace projection. In other words, the interference is often sufficiently strong to corrupt signal reception, but not enough higher than noise to permit effective adaptive cancellation. By adding a few (say one to three) low gain "auxiliary" antennas to an imaging array, it is possible to overcome poor interference parameter

This project is supported by National Science Foundation grant number AST-9987339.

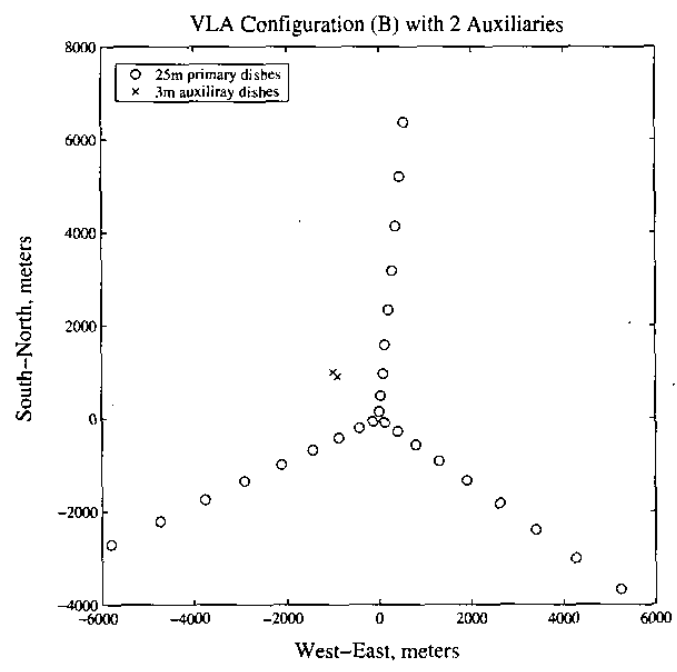

Fig. 1. Array element geometry for the VLA configuration B, showing possible location for two low gain auxiliary $3 \mathrm{~m}$ diameter dishes.

estimation, even with multiple interfering sources. These auxiliaries can, for example, be small dishes steered to track an orbital satellite, or can simply be inexpensive omnidirectional antennas.

This paper discusses and compares four algorithms (described in the following section), including a new approach and extensions to classical and more recently proposed methods. Each of these methods forms a linear transform on post-correlator data to produce estimates of the desired array signal covariance matrix with reduced interference bias. The idea is to estimate the linear subspace spanned by the interference in sample array covariance, $\hat{\mathbf{R}}$, and to then form a perpendicular projection operator to remove it. This can be performed as a "post processing" operation on the time averaged array covariance matrix estimate at the correlator output, and therefore does not require real-time processing hardware

As an example, Figure 1 illustrates how the Very Large Array (VLA) in Socorro New Mexico can be augmented with two auxiliary antennas for use with the algorithms described below. The array geometry shown is for the $(B)$ configuration, and the $\times s$ indicate two proposed auxiliary locations. Adding this capability to an existing imaging array could be as simple as using a spare channel in the correlator (e.g., a channel normally used for a tem- 
porarily out-of-service telescope dish) to process the signal from a low-cost omnidirectional antenna. We will show that using a low performance auxiliary antenna can produce dramatic improvement in interference rejection at high interference levels.

\subsection{Signal Model}

Consider an $M$ element imaging antenna array with the $n^{\text {th }}$ complex baseband data sample (across the array) represented by

$$
\begin{aligned}
\mathbf{x}[n] & =\left[x_{1}[n], \cdots, x_{M}[n]\right]^{T}, \\
& =\mathbf{s}[n]+\mathrm{i}[n]+\eta[n],
\end{aligned}
$$

where $\mathbf{s}, \mathbf{i}$, and $\eta$ correspond to desired signal, man-made interference, and noise respectively. The array covariance matrix is given by

$$
\mathbf{R}=E\left\{\mathbf{x}[n] \mathbf{x}^{H}[n]\right\},=\mathbf{R}^{s}+\mathbf{R}^{i}+\mathbf{R}^{\eta},
$$

where ${ }^{H}$ indicates matrix conjugate transpose. In radio imaging, this spatial cross-correlation between array element pairs comprises the crucial image forming information. Elements of the signal-of-interest covariance matrix, $\mathbf{R}^{s}$, correspond to image samples in the frequency domain, with sample locations determined by the physical vector distances (called baselines) between antenna pairs. In the absence of noise or interference, this Fourier relationship is expressed by the visibility function [4],

$$
V(u, v)=\iint I(l, m) A(l, m) e^{-j 2 \pi(u l+v m)} d l d m
$$

where $I(l, m)$ is the desired source intensity image and $A(l, m)$ is the antenna beam response. $\mathbf{R}^{s}$ is formed by sampling $V(u, v)$ at discrete points corresponding to baseline vectors for array element pairings

$$
\left\{\mathbf{R}^{s}\right\}_{(i, k)}=V\left(u_{i k}, v_{i k}\right),\left[u_{i k}, v_{i k}\right]^{T}=\mathbf{r}_{k}-\mathbf{r}_{i},
$$

where $\mathbf{r}_{i}$ and $\mathbf{r}_{k}$ are the two-dimensional position vectors for the $i^{t h}$ and $k^{t h}$ array elements respectively, and $\left\{\mathbf{R}^{s}\right\}_{(i, k)}$ indicates the $i^{\text {th }}$ row, $k^{\text {th }}$ column of $\mathbf{R}^{s}$.

The image synthesis problem consists of estimating $\mathbf{R}^{s}$ for a sufficiently large number of unique baseline position pairs, interpolating from these samples to obtain a set of dense rectangular grid samples for $V(u, v)$, and using the 2-D inverse FFT to solve (3) for $\hat{I}(l, m)$ (where indicates an estimated quantity). $\mathbf{R}^{i}$ can introduce errors in $\hat{\mathbf{R}}^{s}$, and removing this bias is the focus of this paper.

An augmented imaging array consists of $M_{p}$ high gain "primary" telescope antennas and $M_{a}$ low gain "auxiliary" antennas, with $M=M_{p}+M_{a}$. The array sample vector is

$$
\mathbf{x}[n]=\left[\begin{array}{c}
\mathbf{x}_{p}[n] \\
\mathbf{x}_{a}[n]
\end{array}\right],
$$

where $\mathbf{x}_{p}[n]$ is the vector signal from the original high gain primary array, and $\mathbf{x}_{a}[n]$ is the auxiliary sub-array signal. It is assumed that the auxiliary antennas have a much higher interference to signal plus noise ratio (ISNR) than the primaries by either tracking the interferer or having low gain to the desired signal. The augmented array covariance matrix has the following block structure

$$
\mathbf{R}=\left[\begin{array}{ll}
\mathbf{R}_{p p} & \mathbf{R}_{p a} \\
\mathbf{R}_{a p} & \mathbf{R}_{a a}
\end{array}\right],
$$

where for example $\mathbf{R}_{a p}=E\left\{\mathbf{x}_{a}[n] \mathbf{x}_{p}^{H}[n]\right\}$.

\section{ALGORITHMS FOR INTERFERENCE REMOVAL FROM CORRELATION DATA}

In this section, several algorithms are described which use only the array sample covariance matrix, $\hat{\mathbf{R}}$, at the correlator processor output to construct reduced interference estimates of the desired signal covariance, $\mathbf{R}^{s}$. In each algorithms a spatial filtering, or projection matrix, $\mathbf{P}$, is derived from $\hat{\mathbf{R}}$ and used to block the interference component, $\mathbf{R}^{i}$. The filtered signal covariance estimate is given by $\hat{\mathbf{R}}^{s}=\mathbf{P} \hat{\mathbf{R}} \mathbf{P}^{H}$, where $\mathbf{P}$ is designed to be orthogonal to $\mathbf{R}^{i}$ but to produce minimal distortion to the signal and noise subspaces. For independent noise, $\mathbf{R}^{\eta}$ is diagonal, and does not seriously affect synthesis imaging which relies on the off-diagonal terms of $\hat{\mathbf{R}}^{s}$

In the discussion below it is assumed (unrealistically) that interferers exhibit no motion relative to the array. The short-term integration and bias removal method described by Raza et al [2] can be applied to all algorithms below to deal with non-stationary interference.

\subsection{Subspace Projection Without Auxiliaries (SP)}

Leshem and van der Veen [1] and Raza et al [2] used a spatial filtering approach to project out the covariance subspace corresponding to interference. The method is summarized here as background for the comparative analysis to follow. As originally described, only the primary data, $\mathbf{x}_{p}[n]$, from high gain antennas is used.

The interference component of $\mathbf{R}_{p p}$ spans a $Q$ dimensional subspace, where $Q$ is the number of distinct interferers present. The desired spatial filter is the perpendicular (relative to this interference subspace) projection matrix, $\mathbf{P}_{\mathrm{SP}}$, i.e. the rank $\left(M_{p}-\right.$ $Q$ ) projector that satisfies $\mathbf{P}_{\mathrm{SP}} \mathbf{R}_{p p}^{i}=\mathbf{0}$. In most cases detailed knowledge of interferer directions is not available, and the array is not precisely calibrated, so $\mathbf{P}_{\mathrm{SP}}$ must be estimated from $\hat{\mathbf{R}}_{p p}$. If the ISNR $>>1$, this can be done by partitioning its eigenvectors,

$$
\begin{aligned}
\hat{\mathbf{R}}_{p p} \mathbf{U} & =\mathbf{U} \boldsymbol{\Lambda}, \\
\mathbf{U}^{s} & =\left[\mathbf{u}_{Q+1}, \cdots, \mathbf{u}_{M_{p}}\right], \\
\mathbf{P}_{\mathrm{SP}} & =\mathbf{U}^{s}\left(\mathbf{U}^{s}\right)^{H},
\end{aligned}
$$

where the eigenvalues in diagonal matrix $\boldsymbol{\Lambda}$ are sorted in descending magnitude, and $\mathbf{U}$ is unitary. The signal covariance estimate is given by $\hat{\mathbf{R}}_{S P}^{s}=\mathbf{P}_{S P} \hat{\mathbf{R}}_{p p} \mathbf{P}_{S P}$.

\subsection{Subspace Projection with Auxiliary Antennas (SPA)}

In this approach, the subspace projection method described above is applied with minor modification to the full augmented array, including auxiliaries. The only special handling required is that we do not wish the cross correlations between primary and auxiliary antennas to appear in the final signal covariance estimate. These correlations have higher noise levels and contain no signal information. The eigenvectors of the full array sample covariance are partitioned, and the filter matrix, $\mathbf{P}_{\mathrm{SPA}}$, is formed as

$$
\begin{aligned}
\hat{\mathbf{R} U} & =\mathbf{U} \mathbf{\Lambda} \\
\mathbf{U}^{s} & =\left[\mathbf{u}_{Q+1}, \cdots, \mathbf{u}_{M}\right], \\
\mathbf{P}_{\mathrm{SPA}} & =\mathbf{I}_{M_{p}} \mathbf{U}^{s}\left(\mathbf{U}^{s}\right)^{H}, \\
\mathbf{I}_{M_{p}} & =[\mathbf{I}, \mathbf{0}],: M_{p} \times M .
\end{aligned}
$$


where $\mathbf{I}_{M_{p}}$ is a truncated identity matrix used to remove the last $M_{a}$ rows in computing $\mathbf{P}_{\mathrm{SPA}}$. Note that $\mathbf{P}_{\mathrm{SPA}}$ is not square, and therefore is not strictly a projection matrix. The covariance estimate is given by $\hat{\mathbf{R}}_{\mathrm{SPA}}^{s}=\mathbf{P}_{\mathrm{SPA}} \hat{\mathbf{R}} \mathbf{P}_{\mathrm{SPA}}^{H}$.

\subsection{Array Multiple Sidelobe Canceller (MSC)}

The classical multiple sidelobe canceller (MSC) [5] can be extended to form a projection-matrix-like operator that can be applied directly to $\hat{\mathbf{R}}$. The MSC adaptive beamformer weight for the $m^{t h}$ primary antenna is given by

$$
\begin{aligned}
y_{m}[n] & =x_{p, m}[n]-\mathbf{w}^{H} \mathbf{x}_{a}[n], ; 1<m<M_{p}, \\
\mathbf{w} & =\mathbf{R}_{a a}^{-1}\left\{\mathbf{R}_{a p}\right\}_{(\text {column } m)} .
\end{aligned}
$$

Using the same auxiliary array in each case, a separate MSC beamformer can be computed for every primary element to produce an array MSC, which is given in matrix form by

$$
\mathbf{y}[n]=\left[\mathbf{I},-\mathbf{R}_{p a} \mathbf{R}_{a a}^{\dagger}\right] \mathbf{x}[n],
$$

where $\mathbf{y}[n]=\left[y_{1}[n], \cdots, y_{M_{p}}[n]\right]^{T}$, and $\mathrm{I}$ is $M_{p} \times M_{p}$. The pseudo inverse is used here to improve numerical stability. The array MSC covariance matrix is given by

$$
\begin{aligned}
\mathbf{R}_{y y} & =\mathbf{P}_{\mathrm{MSC}} \mathbf{R} \mathbf{P}_{\mathrm{MSC}}^{H}, \text { where } \\
\mathbf{P}_{\mathrm{MSC}} & =\left[\mathbf{I},-\mathbf{R}_{p a} \mathbf{R}_{a a}^{\dagger}\right],
\end{aligned}
$$

It can be shown that $\mathbf{R}_{y y} \approx \mathbf{R}^{s}$, so we define $\hat{\mathbf{R}}_{\mathrm{MSC}}^{s}=\mathbf{P}_{\mathrm{MSC}} \hat{\mathbf{R}} \mathbf{P}_{\mathrm{MSC}}^{H}$.

\subsection{Auxiliary Assisted Cross Subspace Projection (CSP)}

It can be argued that the two previous algorithms suffer from structural problems which unnecessarily introduce error in $\hat{\mathbf{R}}^{s}$. Low ISNR correlations in $\mathbf{R}_{\mathfrak{p p}}$ were included in computing the projection/filter matrices. This increases interference subspace estimation error. Also, $\hat{\mathbf{R}}^{s}$ was formed as the product of the filter matrix $\left(\mathbf{P}_{\text {SPA }}\right.$ or $\left.\mathbf{P}_{\mathrm{MSC}}\right)$ and the full $\hat{\mathbf{R}}$. This can unnecessarily re-introduce interference if the spatial filter is not perfect because $\hat{\mathbf{R}}$ includes high interference level terms. The auxiliary assisted cross subspace projection algorithm presented here avoids both of these problems.

The projection matrix is constructed from a partitioned singular value decomposition of cross correlation, $\hat{\mathbf{R}}_{p a}$,

$$
\begin{aligned}
\hat{\mathbf{R}}_{p a} & =\mathbf{U} \Sigma^{\frac{1}{2}} \mathbf{V}^{H} \\
\mathbf{U}^{s} & =\left[\mathbf{u}_{Q+1}, \cdots, \mathbf{u}_{M_{p}}\right] \\
\mathbf{P}_{\mathrm{CSP}} & =\mathbf{U}^{s}\left(\mathbf{U}^{s}\right)^{H}
\end{aligned}
$$

where $\Sigma^{\frac{1}{2}}$ is diagonal and $\mathbf{U}$ and $\mathbf{V}$ are unitary. Note that since noise and signal are far below interference levels in the auxiliary channels, the interference subspace of $\hat{\mathbf{R}}_{p a}$ can be identified with less estimation error. The signal covariance estimate is computed as $\hat{\mathbf{R}}_{\mathrm{CSP}}^{s}=\mathbf{P}_{\mathrm{CSP}} \hat{\mathbf{R}}_{p p} \mathbf{P}_{\mathrm{CSP}}^{H}$. Only primary channel correlations, $\hat{\mathbf{R}}_{p p}$, rather than the full $\hat{\mathbf{R}}$, are operated on by the projection matrix, thus eliminating leakage of interference from the auxiliaries into $\hat{\mathbf{R}}_{\text {CSP }}^{s}$. $\mathbf{P}_{\text {CSP }}$ is shown below to have the best overall performance of the four algorithms.

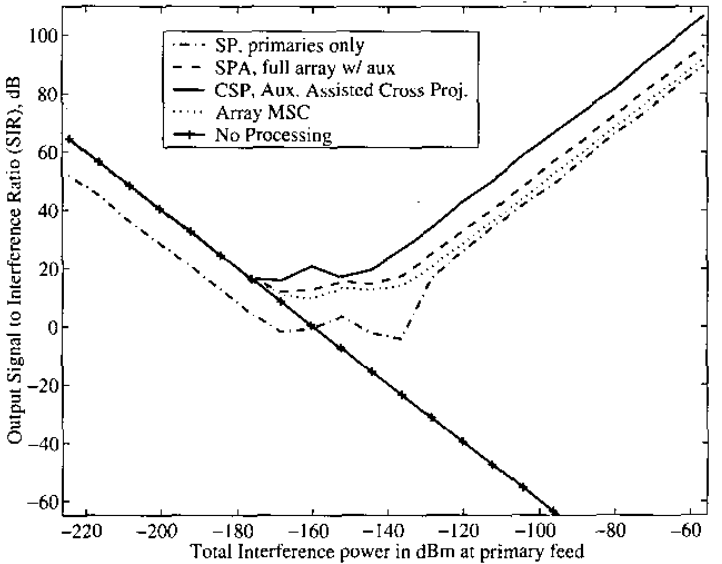

Fig. 2. Signal to interference ratio for two stationary interferers and two small auxiliary dish antennas aimed at the interferers Source is $1 \mathrm{Jy} \mathrm{OH}$ emission.

\section{SIMULATION RESULTS}

This section presents a series of simulations, for two different signal scenarios, to compare interference removal performance for the four algorithms described above. In all cases, The modeled imaging telescope array is the VLA in the configuration of Figure 1. The desired source is a hydroxyl ion $(\mathrm{OH})$ emission at 1612 $\mathrm{MHz}$, and interference is modeled as the spectral sidelobes of a GLONASS satellite transmission. Processing bandwidth is 4.0 $\mathrm{kHz}$ and source bandwidth is $1.0 \mathrm{kHz}$. The number of interferers, $Q$, is assumed known.

Realistic models were used for antenna sidelobe response patterns in magnitude and phase, pattern variation among antennas, interfering satellite orbital motion and signal levels, correlation estimation error for the integration interval, system noise temperature, aperture efficiency, and other parameters required to produce simulation results representative of real experiments. A system noise temperature of $90 \mathrm{~K}$ was assumed for the inexpensive auxiliary channels, as compared with the L-band specification of $35 \mathrm{~K}$ for the $25 \mathrm{~m}$ high gain dishes at the VLA. In the moving interference simulation example, short-time integration as described in [2] was employed for covariance estimation, projection computation, and projection bias removal.

In the following plots, output signal to interference ratio is computed as

$$
\operatorname{SIR}=\frac{\operatorname{Tr}\left\{\frac{1}{K} \sum_{k=0}^{K-1} \mathbf{P}_{k} \mathbf{R}^{s} \mathbf{P}_{k}^{H}\right\}}{\operatorname{Tr}\left\{\frac{1}{K} \sum_{k=0}^{K-1} \mathbf{P}_{k} \mathbf{R}^{i}(\bar{k}) \mathbf{P}_{k}^{H}\right\}},
$$

where $\operatorname{Tr}\{\}$ is matrix trace, $\mathbf{R}^{s}$ the true signal covariance, and $\mathbf{R}^{i}(\bar{k})$ is the true interference covariance at a time snapshot midway through short-term integration interval $k$. For stationary interference cases, $K=1$, and $\mathbf{R}^{i}(\bar{k})$ is constant.

Figure 2 presents results for a scenario with two stationary interferers and two $3 \mathrm{~m}$ dish auxiliary antennas aimed at these satellites. Auxiliary antenna locations are as shown in Figure 1. For 


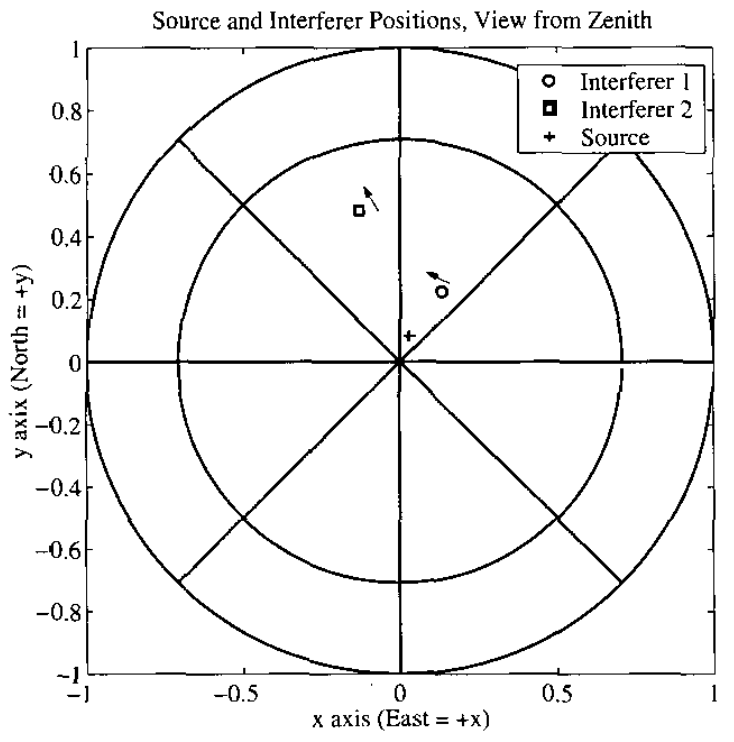

Fig. 3. Positions and motion paths for a simulated $\mathrm{OH}$ radio source and two GLONASS interferers. Zenith is at the center of the graph, inner circle is $45^{\circ}$ elevation.

this case, the $\mathrm{OH}$ source has a flux density of $1 \mathrm{Jy}$ ( 1 Jansky $=10^{-26} \mathrm{~W} / \mathrm{m}^{2} / \mathrm{Hz}$ ), and is located at $20^{\circ}$ azimuth (AZ), $85^{\circ}$ elevation (EL). The interferers are at $30^{\circ} \mathrm{AZ}, 75^{\circ} \mathrm{EL}$., and $-5^{\circ}$ $\mathrm{AZ}, 60^{\circ} \mathrm{EL}$, with the second interferer transmission level $20 \mathrm{~dB}$ below the first. Integration time is 10 seconds. Note that all algorithms using auxiliary antennas outperform the primaries only $\mathrm{SP}$ method. For interference power greater than $-160 \mathrm{dBm}$, all adaptive algorithms yield dramatic improvement over no processing. The CSP algorithm performs best overall for the widest range of interference power levels, while the other algorithms would require a detector function to turn them off if no interferer is present.

Figure 4 presents results for the second scenario, which in cludes two moving GLONASS interferers and two $3 \mathrm{~m}$ dish auxiliary antennas separately steered to track these satellites. Auxiliary locations are as shown in Figure 1. The $\mathrm{OH}$ source level is once again $1 \mathrm{Jy}$. Source location and initial interferer locations are as in scenario one. Figure 3 illustrates the relative positions, and orbital track motion vectors. Long-term integration time is 1 second, with short-term integrations of $5 \mathrm{~ms}$. This short interval was required to reduce subspace smearing and obtain acceptable interference rejection given satellite motion. Once again the new CSP algorithm is the overall best performer.

\section{CONCLUSIONS}

The simulations presented above suggest that in most signal and interference regimes, use of high ISNR signals from auxiliary antennas will significantly improve interference removal. Subspace projection techniques perform much better with auxiliaries, and the MSC and CSP algorithms perform well, seamlessly from very low to very high interference levels.

We have already successfully tracked GLONASS and Irid-

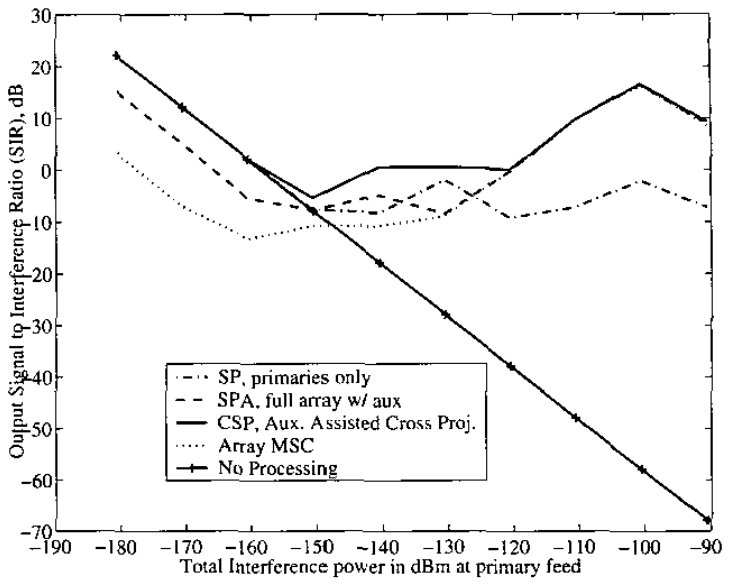

Fig. 4. Signal to interference ratio for two orbiting GLONASS interferers and two $3 \mathrm{~m}$ steered dish auxiliary antennas tracking the satellites. Source is $1 \mathrm{Jy} \mathrm{OH}$ emission.

ium satellite sources and computed real-time array correlations using low cost $3 \mathrm{~m}$ az-el mount dish antennas which are similar to the Small Radio Telescope (SRT) system introduced by MIT Haystack Observatory. Satellite orbital parameters and tracking control commands were generated by a low cost readily available software package, "NOVA for Windows." This suggests that adding such an auxiliary antenna to an existing imaging array would not be prohibitively costly.

\section{REFERENCES}

[1] A. Leshem, A.J, van der Veen, and A.J. Boonstra, "Multichannel interference mitigation techniques in radio astronomy," Astrophysical Journal Supplements, vol. 131, no. 1, pp. $355-374,2000$.

[2] J. Raza, A.-J. Boonstra, and A.-J. van der Veen, "Spatial filtering of $\mathrm{rf}$ interference in radio astronomy," IEEE Signal Processing Letters, vol. 9, no. 2, pp. 64-67, Feb. 2002.

[3] C. Barnbaum and R.F. Bradley, "A new approach to interference excision in radio astronomy: Real-time adaptive cancellation," Astronomical Journal, vol. 116, pp. 2598-2614, Nov. 1998.

[4] A.R. Thompson, J.M. Moran, and G.W. Swenson Jr., Interferometry and Synthesis in Radio Astronomy, Second Edition, Wiley-Interscience, New York, 2001.

[5] S.P. Applebaum and D.J. Chapman, "Adaptive arrays with main beam constraints," IEEE Transactions on Antennas and Propagation, vol. AP-24, pp. 650-662, Sept. 1976. 